\title{
ELECTROCHEMICALLY-BASED DOSE MEASUREMENT FOR CLOSED-LOOP DRUG DELIVERY APPLICATIONS
}

\author{
Christian A. Gutierrez, Roya Sheybani and Ellis Meng \\ University of Southern California, Department of Biomedical Engineering, Los Angeles, CA, USA
}

\begin{abstract}
We present a method for accurate, real-time tracking of drug dose volume in reservoir-based drug pump systems using electrochemical impedance measurements. Detection of physiologically-relevant drug volumes $(500 \mathrm{~nL}-64 \mu \mathrm{L})$ and on-the-fly flow rate variations $(2.78-80 \mu \mathrm{L} / \mathrm{min})$ from a MEMS drug delivery system were demonstrated. The sensor consists of two microelectrodes integrated within the drug reservoir. This method is attractive for its simplicity, sensitivity $(0.2 \Omega / \mathrm{nL})$, and wide drug compatibility. The capability of electronic, real-time dose measurement paves the way for fully-integrated closed-loop drug delivery systems.
\end{abstract}

\section{KEYWORDS}

Drug delivery, electrochemical impedance, dose tracking.

\section{INTRODUCTION}

Controlled administration of drugs can increase drug therapy effectiveness, in some cases up to $60 \%$ [1]. Although growing evidence supports patient-tailored regimens to achieve optimum efficacy, most drug delivery devices are preset to deliver drug at a constant rate [2]. This is in part due to the lack of electronic dosing control in current devices precluding the ability to track, adjust and confirm drug dosage.

Nondestructive pharmacokinetic methods have been developed to track and confirm dosage levels; however these techniques are restricted to unique systems such as direct observation, microdialysis, nuclear imaging and blood measurements but suffer from significant limitations in their resolution, accuracy, and/or detection limits. Furthermore, these methods cannot provide realtime feedback and rely on indirect indicators to confirm dosage. Integration of more traditional fluid monitoring techniques, such as flow sensor technologies, presents a significant packaging challenge and can be rendered ineffective under the low volume/flow dosage regimens often required by implantable devices. A direct and realtime method to track and confirm delivered volume based on electrochemical impedance measurements from a fully-integrated drug delivery micropump (Figure 1) is presented here.

We previously demonstrated the ability to track subnanoliter liquid volumes, encapsulated within Parylenebased microstructures, through electrochemical impedance measurements [3]. This technique offered a straightforward, highly accurate method to realize real-

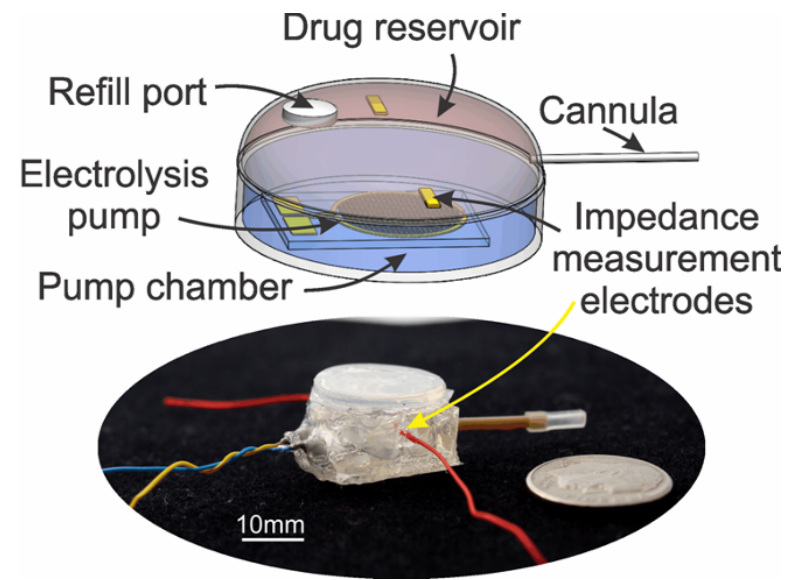

Figure 1: (Top): Model of drug delivery system, (bottom): actual integrated device

time volume tracking. Based on this work, we apply electrochemical impedance measurements to tracking larger volumes relevant for drug delivery applications.

Previous attempts to monitor drug dosing utilized impedance-based measurements of the gas/liquid fraction within an electrolysis chamber [4]. Active pumping was demonstrated but utilized rigid materials (silicon/Pyrex ${ }^{\circledR}$ ) in a format not well suited to implantable applications. This approach was also limited to small drug delivery volumes $(<1.5 \mu \mathrm{L})$ and would not be appropriate for sustained long-term use. The ability to accurately monitor and control delivered volume to within $\pm 5 \mathrm{~nL}$ was, however, achieved. Another approach demonstrated the ability to monitor the dissolution rate of reservoirs containing 35-45 $\mu \mathrm{g}$ of drug (in solid form) upon exposure to a liquid $(1 \times \mathrm{PBS})$ environment [5]. The lack of active control over the delivery profile is a drawback to this approach which relies solely on passive diffusion processes to distribute the drug. For sustainedrelease applications, however, this method provided the first demonstration of non-invasive, real-time monitoring of drug dissolution rate.

In this work, we present real-time tracking and accurate monitoring of a wide range of drug dosage volumes by electrochemical impedance measurements in a fully-integrated drug delivery system featuring active electrolysis-based pumping [6].

\section{DEVICE DESIGN AND OPERATION Pump Construction}

Pump operation is based on electrolytically generated pneumatic actuation. Hydrogen and oxygen gas are generated by electrolysis within a pump chamber initially filled with water. The pressure generated within 


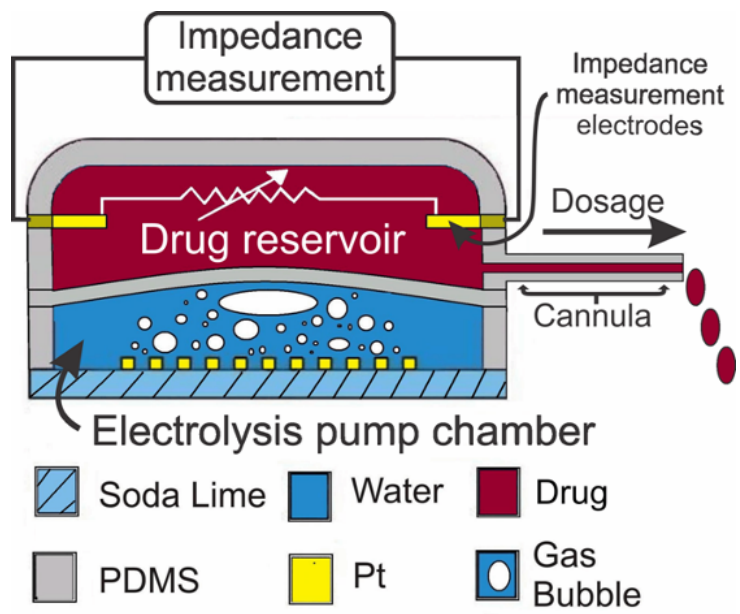

Figure 2: Device cross-section demonstrating impedance measurement and electrolysis-based actuation.

the pump chamber actuates a thin diaphragm that then displaces liquid contained within the attached drug chamber. This results in outflow through an integrated cannula and delivery of the drug (Figure 2).

Interdigitated electrolysis pump electrodes ( $\mathrm{Ti} / \mathrm{Pt}$ $300 \AA / 2000 \AA, 100 \mu \mathrm{m}$ width/spacing) were fabricated on a soda lime substrate by liftoff and then potentiostatically cleaned at $\pm 0.5 \mathrm{~V}$ in $1 \times$ phosphate buffered saline. Nafion ${ }^{\circledR}$ was applied to the electrodes by dip coating two times $(1 \mu \mathrm{m}$ thick coating) to improve gas generation efficiency. The remaining parts were produced by replica casting of polydimethylsiloxane (PDMS) from conventionally machined acrylic master molds.

To assemble the pump, first, electrolysis electrodes were adhered on the pump chamber base. Then the pump diaphragm (400 $\mu \mathrm{m}$ thick) and drug reservoir were attached using PDMS prepolymer and cured in place. Impedance measurement electrodes were then integrated into the drug reservoir. Two dedicated impedance measurement microelectrodes (standard solid 30 AWG wire) were inserted and attached to the reservoir using PDMS prepolymer and cured in place. Finally, the pump chamber and reservoir were filled with deionized (DI) water (serving as electrolyte and model drug, respectively) using a syringe.

\section{Electrochemical Impedance Measurements}

Electrochemical impedance is measured through application of a small alternating current across the electrodes. The electrode-electrolyte interface is modeled by the Randles circuit which consists of the solution (electrolyte) resistance in series with the parallel combination of the double layer capacitance and polarization resistance. By applying a sufficiently high frequency $(>1 \mathrm{kHz})$, the impedance response is dominated by the solution resistance. Consequently, the impedance response can be approximated as a simple classical resistance that is inversely proportional to the total cross-sectional area of fluid between the electrodes and is directly analogous to a variable "fluidic" resistor.
This phenomenon enables the tracking of volume within the drug reservoir.

Electrochemical impedance measurements can be performed with very little power $(<100 \mu \mathrm{W})$ and produce only reversible electrochemical species. Hydrolysis of the electrolyte does not occur so long as the magnitude of the applied AC signal is maintained within the "water-window" of the electrode material; this value is typically greater than $1 \mathrm{~V}$. Details of the electrochemical interface and associated phenomena have been studied in our previous work [7] and have also been thoroughly reviewed in the electrochemistry literature [8].

\section{EXPERIMENTAL METHODS}

The drug reservoir was filled with DI water which represents an aqueous drug solution. Direct delivery of drug to a targeted location can provide therapeutic effects with smaller doses or more dilute formulations when compared to systemic routes. Here, DI water provides an analog for an aqueous drug dilution.

Electrolysis-based actuation of the pump diaphragm was accomplished by applying varying levels of stimulation current between the interdigitated pump chamber electrodes. Current levels ranging between 1-10 $\mathrm{mA}$ were applied to generated pneumatic pressure within the pump chamber, thereby displacing the diaphragm upwards into the drug chamber. The displaced liquid in the drug reservoir was then ejected via the attached cannula. For large delivery volumes $(>10 \mu \mathrm{L})$, the ejected fluid was collected and measured by weight using a precision balance. For low delivery volumes $(<$ $10 \mu \mathrm{L}$ ), the delivery cannula was attached to a calibrated micropipette in which delivery volume was tracked optically by recording the displacement distance of the liquid front within the micropipette.

Impedance measurements were acquired in real-time via a LabVIEW-interfaced precision LCR meter $\left(1 \mathrm{~V}_{\mathrm{pp}}\right.$, $5 \mathrm{kHz}$ ) connected to the impedance measurement electrodes. At this voltage level, only completely reversible chemical processes took place at the electrodes and no chemical modification of the drug occurred. Impedance measurements were acquired throughout each experiment without any additional signal conditioning or filtering.

\section{RESULTS AND DISSCUSION}

Two-electrode impedance spectroscopy was performed between $0.1-100 \mathrm{kHz}$ to determine the frequency range over which solution resistance dominates the impedance response (Figure 3). A $5 \mathrm{kHz}$ measurement frequency was selected. This value is sufficient to bypass the double layer electrode capacitance while avoiding parasitic effects encountered at higher frequencies. 


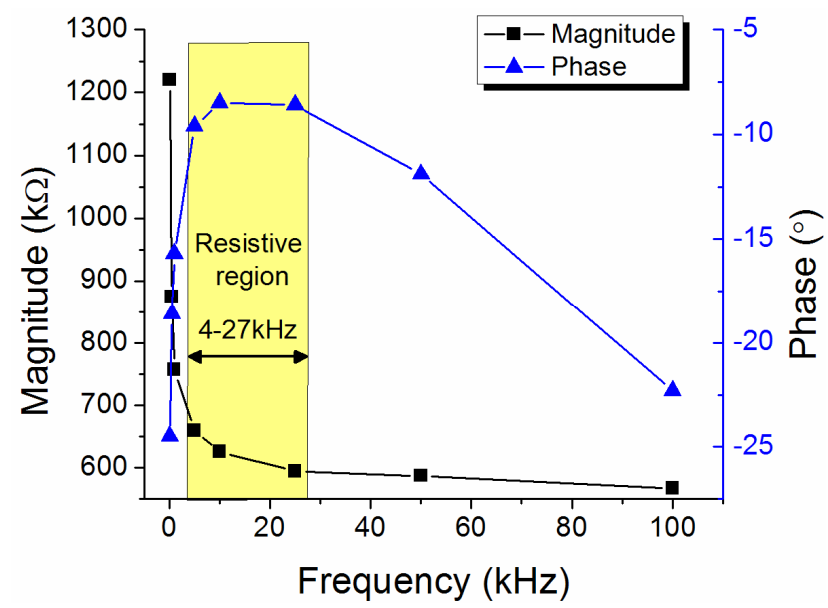

Figure 3: Two-electrode electrochemical impedance spectroscopy of electrode/reservoir system indicating resistive region where solution resistance dominates and capacitive effects are minimized.

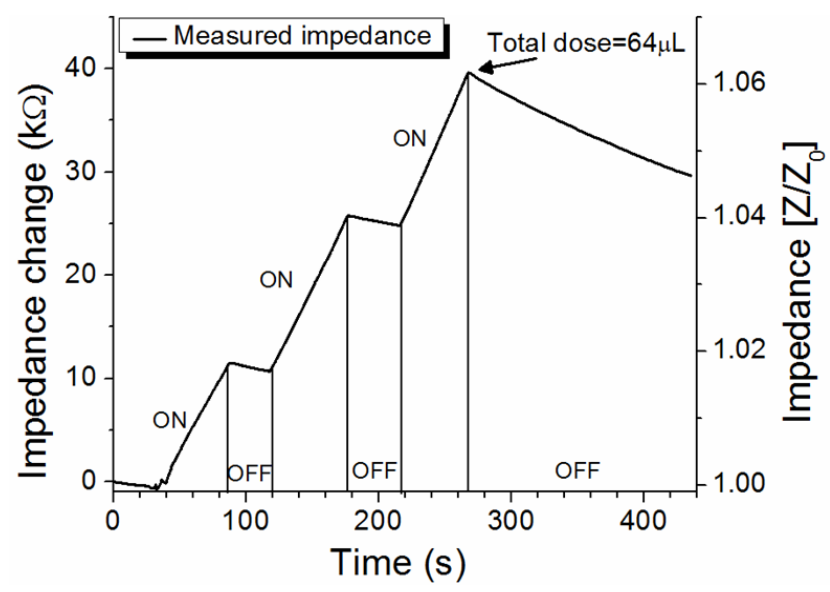

Figure 4: Detection of pump ON/OFF states at $1 \mathrm{~min} / 30 \mathrm{sec}$ intervals respectively. Total delivery volume was $64 \mu \mathrm{L}$ at a pump current of $5 \mathrm{~mA}$.

\section{ON/OFF State Detection}

The ability to detect pump ON/OFF states was first evaluated. A $5 \mathrm{~mA}$ pump current was applied during the ON condition and no current was applied in the OFF condition. Consecutive ON/OFF states were applied in $1 \mathrm{~min} / 30 \mathrm{sec}$ intervals, respectively, while impedance was continuously monitored (Figure 4). Pump ON states were readily detected by an immediate and sustained increase in the impedance magnitude. Immediately after turning the pump off, a sustained decrease in the impedance magnitude was observed. This sustained decrease is a combined result of the restorative elastic forces of the pump diaphragm and the recombination of gases that occurs in the OFF state. The result is a pullback effect that restores the drug chamber to its resting volume and consequently reduces the measured impedance. A total dose of $64 \mu \mathrm{L}$ ( $8 \%$ of reservoir) was produced by the combined 3 minutes of pump activation. A corresponding increase of $6 \%$ was observed in the impedance magnitude over baseline.

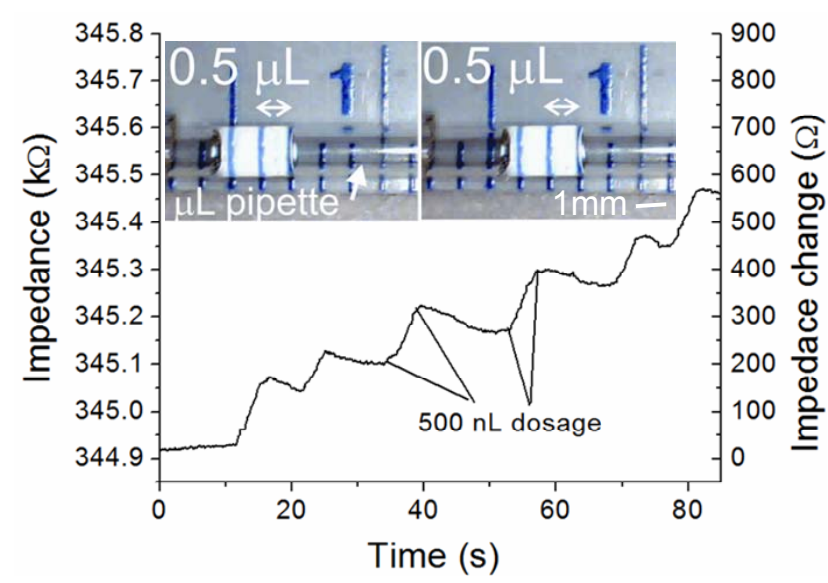

Figure 5: Detection of $500 n L$ bolus delivery as measured by calibrated micropipette. Pump is activated with $1.5 \mathrm{~mA}$ current in step-wise fashion. Measured detection sensitivity of $0.2 \Omega / n L$ was achieved using impedance-based technique.

\section{Tracking of Sub-Microliter Delivery Boluses}

Discrimination of sub-microliter delivery volumes was possible and demonstrates the sensitivity of this method. Delivery of $500 \mathrm{~nL}$ boluses $(0.625 \%$ of reservoir) was measured by impedance and confirmed using a calibrated micropipette $(50 \mu \mathrm{L})$. Boluses were generated by applying $1.5 \mathrm{~mA}$ current pulses (3.4 seconds). Corresponding impedance variations of approximately $150 \Omega$ were observed per delivery bolus and provided a very clear, robust impedance-based measurement (Figure 5). A sensitivity of $0.2 \Omega / \mathrm{nL}$ was obtained in this mode of delivery. The ability to track even smaller volumes with this technique was already demonstrated by our group and others, however, limitations imposed by the experimental setup and compliance within the pump/reservoir system present challenges to achieving similar detection resolution. Further improvement in detection limit is expected for pumps having rigid drug reservoir walls.

\section{Real-time Tracking of Flow Rate Variations}

Lastly, discrimination of on-the-fly changes in delivery rate (and hence flow rate) was demonstrated. Calibrated flow rate measurements were first obtained for pump currents of $10 \mathrm{~mA}, 5 \mathrm{~mA}$ and $1 \mathrm{~mA}(80$ $\mu \mathrm{L} / \mathrm{min}, 44 \mu \mathrm{L} / \mathrm{min}$, and $2.78 \mu \mathrm{L} / \mathrm{min}$, respectively). These three pump current levels were then applied consecutively for 30 second durations and with transitions occurring in real-time while impedance was monitored. A clear variation in the slope of the monotonically increasing impedance magnitude was observed and directly corresponded to changes in the applied pump current levels.

The time-derivative of the measured impedance signal was plotted and yielded distinct levels for each applied current and clearly indicated tracking of their 


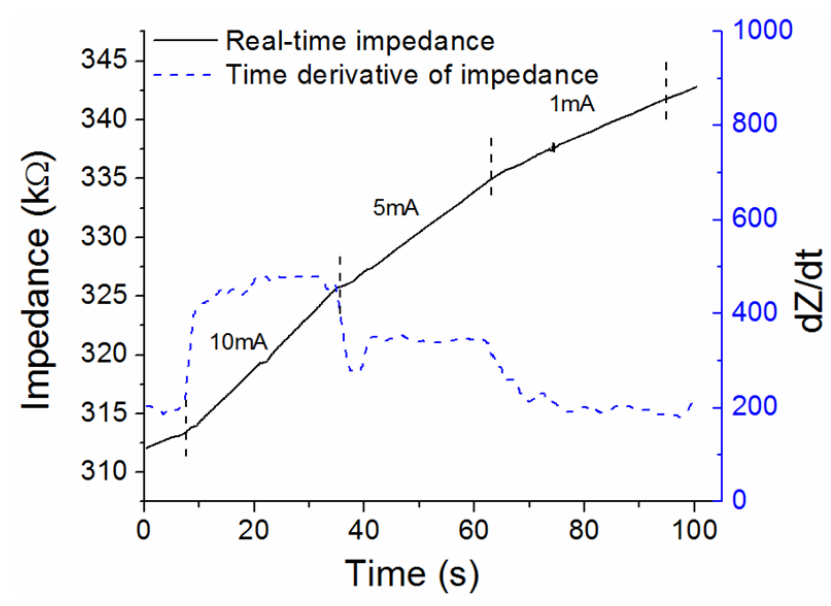

Figure 6: Measurement of on-the-fly flow rate variations at different pump current levels. $10 \mathrm{~mA}=80 \mu \mathrm{L} / \mathrm{min}$, $5 \mathrm{~mA}=44 \mu \mathrm{L} / \mathrm{min}$ and $1 \mathrm{~mA}=2.78 \mu \mathrm{L} / \mathrm{min}$.

transitions, $(3.4 \Omega / \mathrm{s}$ per $\mu \mathrm{L} / \mathrm{min}$ change in flow rate, Figure 6). This procedure allowed for the discrimination and measurement of the rate of delivered volume and thus delivery flow rate. A maximum flow rate measurement of $80 \mu \mathrm{L} / \mathrm{min}$ was demonstrated here but a wider range is possible by applying greater current levels to the electrolysis pump. Current levels up to $13 \mathrm{~mA}$ have been typically utilized to generate high throughput flow rates in excess of $100 \mu \mathrm{l} / \mathrm{min}$. Current device construction and design may impose limitations to the maximum achievable flow rates but their effect was not studied here.

Closed-loop operation based on electrochemical impedance feedback can be easily realized in a complete system. Both delivered volume and flow rate can be measured and utilized as control parameters for calibrated, real-time adjustments to pump inputs, namely the magnitude of applied pump current. Impedancebased feedback enables for the first time real-time control and confirmation of physiologically-relevant delivery volumes in a fully integrated, active drug pump system.

\section{CONCLUSION}

Measurement of physiologically relevant volumes as well as on-the-fly flow rate changes using electrochemical impedance in a MEMS electrochemical pump was demonstrated as a promising technique for closed-loop drug delivery. This method is attractive for its simplicity, sensitivity, wide-compatibility and serves as an important step towards fully-integrated closed-loop drug delivery systems.

\section{ACKNOWLEDGEMENTS}

This work was funded in part by Wallace H. Coulter Foundation Early Career Translational Research Award and the Bill and Melinda Gates Foundation (CG). An OAI Model $30 \mathrm{UV}$ light source was used in the fabrication of the electrodes featured in this work. The authors also would like to thank Dr. Donghai Zhu and the members of the USC Biomedical Microsystems Laboratory for their assistance.

\section{REFERENCES:}

[1] B. Bruguerolle and G. Labrecque, "Rhythmic pattern in pain and their chronotherapy," Adv. Drug Deliv. Rev., vol. 59, pp. 883-895, 2007.

[2] L. Peppas-Brannon, "Recent advances on the use of biodegradable microparticles and nanoparticles in controlled drug delivery," Int J. Pharm., vol. 116, pp. 1-9, 1995.

[3] C. A. Gutierrez and E. Meng, "Improved SelfSealing Liquid Encapsulation in Parylene Structures by Integrated Stackable Annular-Plate Stiction Valve," in IEEE MEMS. Hong Kong, China, 2010, pp. 524-527.

[4] S. Bohm, B. Timmer, W. Olthuis, and P. Bergveld, "A closed-loop controlled electrochemically actuated micro-dosing system," J. Micromech. Microeng, vol. 10, pp. 498-504, 2000.

[5] A. M. Johnson, D. R. Sadoway, M. J. Cima, and R. Langer, "Design and Testing of an Impedance-Based Sensor for Monitoring Drug Delivery," J. Electrochem. Soc., vol. 152, pp. H6-H11, 2005.

[6] P.-Y. Li, R. Sheybani, C. A. Gutierrez, J. T. W. Kuo, and E. Meng, "A Parylene Bellows Electrochemical Actuator," J. Microelectromech. Syst., vol. 19, pp. 215-228, 2010.

[7] C. A. Gutierrez and E. Meng, "Parylene-based Electrochemical-MEMS Transducers," J. Microelectromech. Sys., vol. 19, pp. 1352-1361, 2010.

[8] D. Erickson and D. Li, "Integrated microfluidic devices," Analytica Chimica Acta, vol. 507, pp. 1126, 2004.

\section{CONTACT}

C.A. Gutierrez, tel: 213-821-3949; cagutier@usc.edu 